\title{
Ein Quantum Trost
}

\section{A Quantum of Solace}

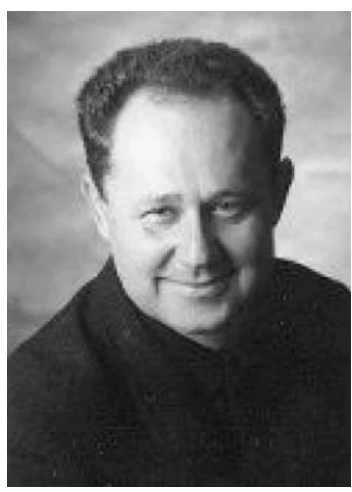

Prof. Dr. Manfred Wildner
Bibliografie

DOI 10.1055/s-0029-1215557

Gesundheitswesen 2009;

71: 205-206

(c) Georg Thieme Verlag KG

Stuttgart · New York

ISSN 0941-3790

Korrespondenzadresse

Prof. Dr. med. M. Wildner

Bayerisches Landesamt für

Gesundheit und

Lebensmittelsicherheit

Veterinärstraße 2

85762 Oberschleißheim

manfred.wildner@|gl.bayern.de
Daniel Craig alias James Bond nimmt die Verfolgung der Geheimorganisation „Quantum“ auf. Im Verlauf des 22. Films dieses Agententhrillers (Regie: Marc Forster) verfolgt Bond seinen Gegenspieler Dominic Greene, den verräterischen Leiter einer internationalen Umweltschutzorganisation, bis nach Bolivien. Die Erzählung bietet eine überraschende Wendung: Während der ebenfalls anwesende Geheimdienst einer Supermacht am Zugang zu sich verknappenden Ölvorräten interessiert ist, hat Dominic Greene Interesse an einem scheinbar wertlosen Stück Land. Welches Geheimnis gilt es zu entdecken? Unter der Oberfläche befinden sich große unterirdische Wasservorräte. Greene will dieses Wasser vor dem Hintergrund einer künstlich herbeigeführten Dürre teuer verkaufen. Am Ende wird Greene von Bond mit einer Dose Motoröl in der Wüste ausgesetzt. James Bond im Gesundheitswesen? Der Leser mag angesichts dieser Profanität nach dem gesundheitlichen Bezug fragen, neben dem Verdursten des Ökologie-Terroristen. „It's the economy, stupid“ („Es ist die Ökonomie, klar doch“)! Die kurze Erzählung bietet Zugang zu einigen ökonomischen Begriffen, die auch für das Gesundheitswesen von zentraler Bedeutung sind. Beginnen wir mit dem wirtschaftlichen Gut „Wasser“. Dessen Produktionskosten ergeben sich durch die eingesetzten Ressourcen bei der Gewinnung und Aufbereitung: Arbeit, Boden, Umwelt und Kapital. Diese unterscheiden sich bei Trinkwasser und Mineralwasser in nicht allzu hohem Maß: Eine süddeutsche Großstadt macht damit Werbung, dass ihr Trinkwasser Mineralwasserqualität hat, jedoch einen vielfach günstigeren Preis („eine Kiste Mineralwasser für 1,7 Cent"). Preise orientieren sich an den Marktbedingungen. In freien Marktwirtschaften ergeben sie sich in der Regel aus dem Zusammenspiel von Angebot und Nachfrage: Ist die Nachfrage groß, das angebotene Produkt jedoch knapp, gehen die Preise in die Höhe - und umgekehrt. Der Preis ist dabei überraschend unabhängig von den ursprünglichen Produktionskosten. Dass die Zahlungsbereitschaft der Käufer auch von geschicktem Marketing beeinflusst werden kann, kann in jedem Supermarkt bei den Mineralwässern direkt in Augenschein genommen werden. Es ist anzunehmen, dass die Zahlungsbereitschaft der fiktiv in Wassernot geratenen bolivianischen Bevölkerung theoriegemäß angestiegen wäre - die Differenz zwischen Aufwand und Ertrag wäre als Gewinn beim Öko-Bösewicht Greene verblieben. Es ist weiterhin anzunehmen, dass Greene, in Not geraten, auch ohne Marketing jeden Preis für lebensrettendes Wasser gezahlt hätte.

Was Greene in der Wüste von Bolivien am eigenen Leib erlebte, bezieht sich auf die Frage nach dem Wert von Gütern, hier auf die Differenz von Tauschwert und Gebrauchswert. Während Kosten und Preise von eingesetzten Ressourcen und Marktmechanismen bestimmt werden, ist der Wert eines Produktes von der eingenommenen Perspektive abhängig. Grundsätzlich kann festgehalten werden, dass der Gebrauchswert des Wassers für das menschliche Leben selbst den von Diamanten unendlich übersteigt: Leben ohne Wasser ist nicht möglich, Leben ohne Diamanten bzw. Rohöl sehr wohl. Die Paradoxie ist, dass Kosten und Preise vom Gebrauchswert entkoppelt sind: Gehen Sie zum Wasserhahn und trinken Sie für Bruchteile eines Cents ein beliebiges Quantum dieser unendlich kostbaren Substanz. „Wertparadoxon“ oder auch „Wasser-Diamanten-Paradoxon“ ist der ökonomische Fachbegriff dafür [1]. An Greenes Situation lässt sich auch eindrücklich das Gesetz abnehmenden Grenznutzens illustrieren: es ist anzunehmen, dass sein persönlicher Nutzen bei den ersten Gläsern lebensrettenden Wassers enorm hoch gewesen wäre, beim hundertsten eher gering [2].

Ökonomische Überlegungen und Forschungen haben nachhaltig Einzug in das Gesundheitswesen gehalten. Sie berühren gleichermaßen die ambulante Vorsorge (Stichwort: Hausarztzentrierte Versorgungssysteme) und die stationäre Versorgung (Stichwort: DRG-basierte prospektive pauschalierte Vergütung), die Akutmedizin genauso wie Pflege, Prävention und Rehabilitation. Das Beispiel „Wasser“ stimmt nachdenklich. Sind menschliche Zuwendung und professionelle Ethik der Heil- und Hilfsberufe womöglich dem Wasser vergleichbar - bildlich gesprochen: mit der „Wasserversorgung“ während der „Wüstenwanderung“ eines Krankenhausaufenthaltes? Von unendlicher Kostbarkeit, dabei in ihrem Gebrauchswert in keiner DRG-Systematik erfasst? Ist es womöglich wie bei der Trinkwasserversorgung im gesellschaftlichen Interesse, dass dieses Gut wegen seines elementaren Nutzens unter 
den meisten Umständen nicht als Ware nach Marktmechanismen gehandelt werden sollte [3]?

„Rasch steigende Kosten in der gesundheitlichen Versorgung haben in den letzten Jahrzehnten die Anwendung von Geschäftspraktiken in der Medizin mit dem Ziel einer erhöhten Effizienz, besserer Ausgabenkontrolle und verbesserter Qualität ausgelöst“, stellen Hartzband und Groopmann fest [4]. Im renommierten New England Journal of Medicine berichten sie aus der amerikanischen Krankenhauswirklichkeit: von Preisen für jede einzelne Verrichtung, von routinemäßig den Ärzten bzw. Therapeuten überstellten Profit- und Gewinnberichten und von Maßzahlen zur Messung der individuellen Kosteneffektivität. Die Autoren stellen weitergehende Fragen: Sind unerwartete Nebenwirkungen solcher Businessstrategien auf die Versorgungsqualität zu erwarten? Welche Auswirkungen bringt die Fokussierung auf Kosten und Preise z.B. für die Interaktion von Therapeuten mit Patienten und auch von Therapeuten untereinander mit sich? In der Beantwortung dieser Frage weisen sie auf einen wichtigen Aspekt hin: Der Unterscheidung zwischen der Ökonomik marktmäßiger Tauschinteraktionen einerseits und gemeinschaftsorientierter sozialer Interaktionen andererseits [5].

Der Ökonom Kenneth Boulding hat diese Frage einmal in einem Satz auf den Punkt gebracht: Niemand liebt General Motors - warum auch? Boulding wollte mit dieser Formulierung die Existenz von unterschiedlichen Sphären gesellschaftlichen Zusammenlebens deutlich machen. Der Gedanke des Tauschhandels ist beim Autokauf sicher eine adäquate Herangehensweise. Hier mag das Ideal des „Homo oeconomicus“, eines ökonomisch rational seine Interessen wahrnehmendes Menschen, zutreffen. Zumindest vordergründig. Sich von der sozialen Emotion „Liebe“ leiten zu lassen, wäre in diesen Zusammenhängen wohl verfehlt. Andere Menschen zu achten und zu lieben, ist dabei nicht grundsätzlich irrational, jedoch ggf. (tausch-)ökonomisch nicht relevant. Das Gegenteil ist in den sozialen Bezügen der Fall, wie z. B. innerhalb einer Familie: Liebe, Fürsorge und gegenseitige geschenkte bzw. vorgegebene Verbindlichkeiten prägen weitgehend die Interaktionen. Eine kalt kalkulierende tauschwirtschaftliche Rationalität wäre hier fehl am Platz - wobei gelegentlich auch behauptet wird, dass genau diese stattfindet, nur unbewusst.

Sind es unrealistische Träume, wenn angesichts der ökonomischen Realitäten an einer primär von menschlichen Werten geprägten Versorgungskultur festgehalten werden soll? Hartzband und Groopmann berichten, dass sich die Atmosphäre in den amerikanischen Krankenhäusern unter den genannten wirtschaftlichen Vorgaben verändert hat, zu Lasten der gemeinschaftsbezogenen und sozialen Dimensionen. Auch empirische Untersuchungen zeigen, dass Hilfsbereitschaft, Kollegialität und andere traditionell hoch geschätzte Qualitäten der professionellen Interaktionen in der gesundheitlichen Versorgung durch eine Neuausrichtung auf tauschökonomisches Handeln leiden [6].

Welches ist die Medizin, die wir wollen? Für unsere berufliche Rolle als Leistungserbringer, für unsere Kinder, unsere Eltern, uns selbst als Patienten? Für welche Medizin sind wir bereit, eine solidarische Finanzierung mit staatlich geregelten Abgabepflichten außerhalb klassischer Marktmechanismen zu akzeptieren? Wo hat die herkömmliche Ökonomik ihren guten und unverzichtbaren Platz im Gesundheitswesen, wo ihre Grenzen? Eine erweiterte Ökonomik für die Sphäre des sozialen Miteinanders ist denkbar, eingebettet in übergeordnete Systemzusammenhänge, bisher jedoch erst in Ansätzen entwickelt [7,8].

Dominic Greene ist in dem zitierten Film nicht mehr rechtzeitig auf eine rettende soziale Sphäre gestoßen, welche ihm das notwendige Quantum Wasser geboten hätte. Vielleicht liefert uns dies einen Denkanstoß, im Gesundheitswesen über vertraute und scheinbar reichlich verfügbare Güter nachzudenken. In höchstem Maß wertvolle Güter, für die keine Preise ausgehandelt wurden und auch nicht aushandelbar sind: Menschlicher Respekt, Zeit, Ansprache, Zuwendung, (Nächsten-)Liebe, ein Quantum Trost. Die Antwort liegt sicher nicht in einem „Entweder-Oder“ und sicher auch nicht in einer unreflektierten, pauschalen Entwertung ökonomischer Ansätze. Die Ökonomik ist als rationaler Ansatz für ein modernes Gesundheitswesen unverzichtbar. Gefordert sind die Wahrnehmung, die kluge Abgrenzung und ein adäquates Schnittstellenmanagement der unterschiedlichen Sphären, die nur in ihrer Komplementarität ein effizientes und humanes Gesundheitswesen nachhaltig sichern können (§70 SGB V). Die Vorgabe von Qualität in der PatientenInteraktion ist sicher unverzichtbar, ebenso wie Überlegungen zur Kosteneffektivität auf Ebene z.B. eines Krankenhauses. Was stört, ist das fehlplatzierte, allgegenwärtige Eindringen detaillierter betriebswirtschaftlicher Kennziffern in die medizinischen und sozialen Interaktionen.

Mit solchen komplementären Aspekten eines „gesunden“ Gesundheitswesen befassen sich die Beiträge in diesem Heft: mit Arzt-Bewertungsportalen im Internet, mit Fehlversorgung beim adulten ADHS, mit der Zufriedenheit von Ärzten im internationalen Vergleich, mit den ökonomischen und gesundheitlichen Aspekten von Rauchverboten in der Gastronomie. Wir hoffen, dass der Einsatz Ihrer kostbarsten Ressource zum Lesen dieser Beiträge - Ihrer Zeit - für Sie gewinnbringend sein wird!

\section{Literatur}

1 Ricardo D. The Principles of Political Economy and Taxation (1817). Dover Pubn Inc, Dover; 2004

2 Gossen HH. Entwickelung der Gesetze des menschlichen Verkehrs und der daraus fließenden Regeln für menschliches Handeln. 1854

3 Hengsbach F. „Mehr Markt“ macht nicht gesund - Gesellschaftliche Risiken und solidarische Sicherung entsprechen einander. Gesundheitswesen 2008; 70: 339-359

4 Hartzband P, Groopman J. Money and the changing culture of medicine. NEJM 2009; 360: 101-103

5 Clark MS, Mills J. Interpersonal attraction in exchange and communal relationships. J Pers Soc Psych 1979; 37: 12-24

6 Vohs KD, Mead NL, Goode MR. The psychological consequences of money. Science 2006; 314: 1154-1156

7 Boulding KE. A preface to grants economics. The economy of love and fear. Praeger Scientific, New York; 1981

8 Kuhn J. Wenn sich Therapie nicht lohnt: Gesundheit als ökonomisches Optimierungsproblem? Forum Kritische Psychologie 2009; 53: 50-54 